\section{Perioperative respira- tory compliance in children undergoing repair of atrial septal defects}

David L. Shulman MD CCFP,

Frederick A. Burrows MD FRCPC,

Delores J. Poppe RN RDCS,

Jeffrey S. Smallhorn MD FRCPC
Children with atrial septal defects (ASD) have less respiratory compliance (Crs) than normal cohorts. There could be implications for anaesthetic management if these children also have decreased compliance during anaesthesia. To examine the changes in Crs before, during and following surgical correction of the atrial defect, Crs was measured in 29 infants and children, 15 undergoing correction of secundum type atrial septal defects, and a group of 14 children of similar weight undergoing non-thoracic surgery. During sedation, Crs was measured using the single breath technique (SBT) and during anaesthesia, both before and after the surgical procedure, an inflation technique was applied to determine Crs. To investigate the aetiology of the difference in Crs, the pulmonary to systemic flow ratio $(\dot{Q} p: \dot{Q} s)$ was determined using echocardiography during sedation in the ASD patients. During sedation, Crs in the ASD group was $52.7 \pm 19.5 \%$ less than in the control group. The slope of the line of regression of Crs vs height for the ASD group was significantly less $(P<0.05)$ than that of the control group during sedation. However, during anaesthesia, Crs in the $A S D$ group was not significantly different from the control group either before or after surgery. The per cent decrease in Crs during sedation in the children with ASD, in comparison with the control group, did not correlate with the $\dot{Q} p: \dot{Q} s$ ratio of the ASD group $\left(r^{2}=0.012, N S\right)$. We conclude that, in spite of lower Crs during sedation, infants and children with ASD do not

\section{Key words}

ANAESTHESIA: paediatric;

CONGENITAL HEART DISEASE: atrial septal defect; LUNG: compliance.

From the Departments of Anaesthesia and Paediatrics (Division of Cardiology) and the Research Institute, The Hospital for Sick Children, University of Toronto, Toronto, Ontario, Canada M5G 1 X8.

Address correspondence to: Dr. F.A. Burrows, Department of Anaesthesia, The Hospital for Sick Children, 555 University Ave, Toronto, Ontario, Canada M5G 1 X8.

Accepted for publication 9 ih November, 1990. have lower Crs during anaesthesia and cardiopulmonary bypass than normal controls undergoing non-thoracic surgery. The decrease in Crs in the children with ASD during sedation is not related to their $\dot{Q}_{p}: \dot{Q} s$.

Les enfants porteurs d'une communication inter-auriculaire (CIA), ont une compliance respiratoire (Crs) moins grande que la normale. Advenant la persistance de ce phénomène pendant l'anesthésie, it y aurait sans doute lieu d'en tenir compte. Nous avons mesuré la Crs avant, pendant et après la fermeture de l'ostium secundum de 15 enfants (groupe CIA) et avons fait de même chez 14 enfants de poids comparables subissant une intervention chirurgicale en dehors du thorax. On mesurait d'abord la Crs par inspiration simple sous sédation puis, par insufflation sous anesthésie avant et après l'opération. Afin de préciser l'étiologie des anomalies de Crs, nous avons aussi mesuré le ratio du débit sanguin pulmonaire sur le débit sanguin systémique $(\dot{Q} p(\dot{Q} s)$ par échocardiographie chez le groupe CIA. Sous sédation, les enfants du groupe CIA avaient une Crs de $52.7 \pm 19,5 \%$ inférieure à celle des enfants normaux et la pente de la droite de régression de la Crs vs la taille des enfants était significativement moins raide dans le groupe CIA $(P<0,05)$. Pourtant, sous anesthésie, que ce soit avant ou aprés l'opération, les différences entre les deux groupes s' estompaient sous le seuil de détection statistique. Sous sédation, il n'y avait pas de corrélation entre la faiblesse des valeurs de Crs du groupe CIA et le $\dot{Q} p / \dot{Q} s\left(r^{2}=0,012\right)$. Donc il appert que malgré un déficit notable sous sédation, indépendant du $\dot{Q} p / \dot{Q} s$, la Crs des porteurs de CIA pendant l'anesthésie es la circulation extracorporelle soit comparable à celle d' enfants normaux subissant une intervention chirurgicale non-thoracique.

Total compliance of the respiratory system (Crs) is affected by congenital heart disease (CHD) in young children, and Crs has even been used as an index of cardiovascular status in infants and children with $C H D .{ }^{\prime}$ The Crs then could be expected to change over the course of anaesthesia and surgery when the heart lesion is repaired. 
These changes could have implications for the anaesthetic management of infants and children with CHD.

Preoperative respiratory compliance is reported to be less in children with CHD involving intra-cardiac left to right shunting of blood. ${ }^{1-4}$ However, these reports were based on dynamic compliance measurements which depend on the frequency of respiration. This dependence is especially marked in children who have regional inequalities of pulmonary time constants. Respiratory frequency was significantly increased in children with increased left to right shunts, as compared to control groups of normal children in two studies, ${ }^{2,3}$ and this could result in spuriously increased compliance measurements. The use of an oesophageal balloon for compliance measurements in infants, especially while lying supine, has been questioned since the highly compliant chest wall in infants can be distorted during respiratory excursions and result in regional inhomogencities in intra-pleural pressure. ${ }^{5}$ Thus the results of these previous studies must be interpreted with caution.

Reports of intraoperative changes in respiratory compliance associated with repair of congenital heart lesions are conflicting. ${ }^{6-8}$ This is probably due to lack of contemporaneous control groups in most of these studies, ${ }^{7-9}$ and to lack of homogeneity in clinical status of the children in the study group. ${ }^{9}$ In order to obtain a clear picture of perioperative Crs changes in children with $\mathrm{CHD}$, a homogeneous group of study patients must be compared to a contemporaneous control group using a test of compliance which does not rely on standard dynamic compliance measurements.

The single breath test (SBT) is a technique for measurement of total respiratory compliance in the sedated infant or child which can also be applied to children during anaesthesia. ${ }^{10,11}$ By this method Crs has been shown to correlate well with compliance measured using an oesophageal balloon in puppies. ${ }^{12}$ The $\mathrm{Crs}$ is also similar to static respiratory compliance measured by the multiple occlusion technique in infants and children, ${ }^{13}$ and is similar to values of compliance measured by a technique of volume recruitment during anaesthesia. ${ }^{11}$ Because of its simplicity, the SBT can be employed to follow compliance changes serially in the perioperative period in a single patient, thus eliminating the methodological problems cited above.

In this study Crs was measured using the SBT in young children during repair of a secundum-type atrial septal defect (ASD) and their preoperative, intraoperative and postoperative compliance were compared with those from a similar group of cardio-respiratory normal children undergoing non-cardiac surgery.

To investigate the aetiology of the difference in the preoperative Crs we measured the pulmonary to systemic blood flow ratio (Q⿱中一p: $\dot{Q} s)$ by echocardiography in the children with ASD and attempted to correlate the values obtained to the per cent difference in preoperative Crs from the control group.

\section{Methods}

With approval from the Human Ethics Committce, informed written consent was obtained from the parents of 29 children, aged three months to six years. They included 15 children who presented for elective repair of a secundum type atrial septal defect (ASD group). The remaining 14 children, who constituted the control group, were ASA physical status I and scheduled to undergo non-cardiac procedures.

Patients were sedated with oral chloral hydrate 50 $\mathrm{mg} \cdot \mathrm{kg}^{-1}$ and $\mathrm{Crs}$ was measured with the SBT. ${ }^{10}$ After induction of anaesthesia with halothane and intubation facilitated by pancuronium, compliance was measured with an inflation technique. Anaesthesia was maintained with halothane. Muscle relaxation was maintained with pancuronium. After the procedure, compliance was measured again with the inflation technique prior to reversal of the neuromuscular blockade.

\section{Respiratory compliance measurement}

For the single breath test, a pneumotachograph (Fleisch 0) was attached to the anaesthetic mask for measuring flow (V) and its integral, volume (V). The pressure outputs from the pneumotachograph were attached to a transducer (digital pneumotachograph, Vertek VR 4000) and the signals were stored on a computer (Hewlett Packard 2623A). The pressure in the mouth ( $\mathrm{Pm})$ was measured through a port on the anaesthetic mask via non-compliant tubing attached to a transducer (Honeywell 143PC0ID) with the aid of the computer which gave an on-line display of $\dot{V}$ and Pm (Figure 1). During quict breathing at end-inspiration the pneumotachograph was briefly occluded until $\mathrm{Pm}$ reached a plateau at pressure $\mathrm{P}$. The occlusion was then released and tidal breathing resumed. The post-occlusion expiration was displayed as a flowvolume ( $\dot{V}-\mathrm{V})$ curve (Figure 2 ), and the linear segment was chosen by the operator. The least squares regression line of the segment chosen was extrapolated to the volume axis, intersecting it at the relaxed end-expiratory volume of the respiratory system (Vrs). The Crs was the volume of the passive expiration from end-inspiration to Vrs, divided by P. From eight to 12 SBT's were carried out during sedation.

In the inflation technique, a volume of anaesthetic gas equal to the patients' approximate anaesthetized tidal volume, $5 \mathrm{ml} \cdot \mathrm{kg}^{-1}$, was delivered into the tracheal tube with a graduated syringe. The airway was occluded during and after the inflation for three seconds in order to 

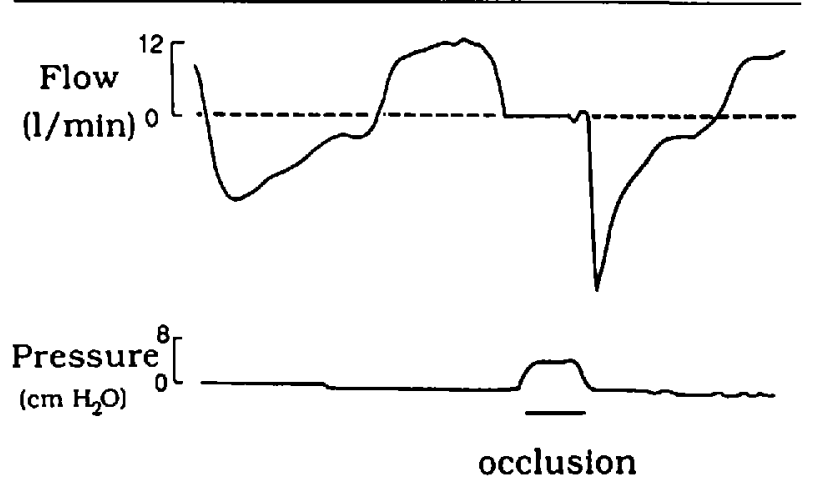

FIGURE I Apparatus for the single-breath test. Flow and pressure signals from the pncumotachograph during the single-breath test were displayed on-line and stored on computer for subsequent analysis.

measure airway pressure at equilibrium. The airway occlusion was then released and ventilation resumed. The delivered volume, corrected to BTPS, divided by the equilibrium pressure, was $\mathrm{Crs}$ which was averaged from six to ten inflations.

\section{Echocardiographic assessment}

Echocardiography was performed during sedation using either an ATL Ultramark 8 (Advanced Technology Laboratories) or an HP 77020A (Hewlett Packard) Ultrasound systems.

Pulmonary valve diameter was measured in systole from the parasternal short axis and aortic valve diameter from the parasternal long axis. Doppler measurements were made utilizing a $3 \mathrm{MHZ}$ transducer across the pulmonary valve from parasternal short axis and across the aortic valve from the apical four-chamber view. A mean Doppler velocity was measured using the Sony Cardiologic Analysis System. The Qp:Q were made using the standard Doppler formula. ${ }^{14-17}$

Due to the absence of tricuspid or pulmonary regurgitation in the study population it was not possible to calculate the systolic pulmonary artery pressure.

\section{Data analysis}

Anthropometric data of the ASD and normal groups were compared using the Mann-Whitney $U$ test. The regression of Crs vs height for the non-cardiac children was accepted as the normal regression line. The slopes and $y$ intercepts of the lines of regression of Crs vs height for the ASD children were compared with these normal regression lines using Student's t test. Time-dependent data were analysed using analysis of covariance and the Tukey multiple range test. Statistical significance was assumed for $P<0.05$.

The per cent difference in Crs was calculated as the difference between Crs for each ASD child and the

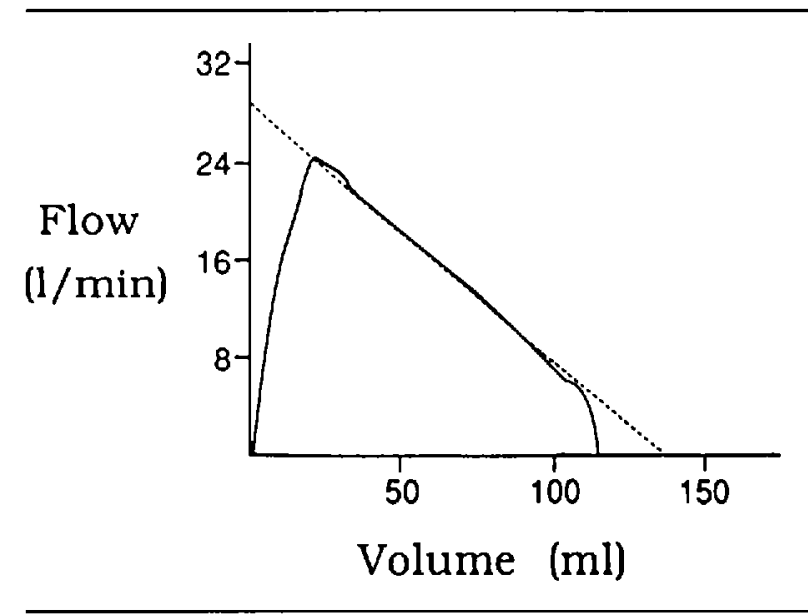

FIGURE 2 Flow-volume ( $\dot{V}-\mathrm{V})$ curve for the post-occlusion cxpiration in the single breath test. The linear segment of the $\dot{V}-V$ curve is extrapolated to intersect the volume axis at the relaxed end-expiratory volume of the respiratory system (Vrs). Total respiratory compliance (Crs) equals the volume of passive expiration from endinspiration to Vrs divided by $P$, the plateau pressure measured at the mouth during occlusion.

expected Crs for a normal child from the normal regression line at that height. The difference was then expressed as a percentage decrease from the expected Crs.

The relationship between the Qि: $\dot{Q} s$ ratio and the per cent decrease in Crs was analysed by least squares linear regression and the correlation expressed as the coefficient of determination $\left(r^{2}\right)$.

\section{Results}

There was no significant difference in the anthropometric data between the two groups of patients (Table).

During sedation, the mean difference in Crs of the ASD group from the expected Crs was $-52.7 \pm 19.5 \%$ and the slope of the line of regression of $\mathrm{Crs}$ versus height for the ASD group was significantly less than that of the control group (Figure 3). However, both after the induction of anaesthesia and after surgery the slopes and $y$ intercepts of the line of regression of Crs vs height for the ASD and control groups were significantly less than during sedation, but the ASD group was no longer different from the control group.

Analysis of echocardiographic and Doppler assessment of $\dot{\mathrm{Q}}: \dot{\mathrm{Q}} \mathrm{s}$ revealed no correlation between the per cent

TABLE Anthropometric data

\begin{tabular}{lllll}
\hline & Number & Weight $(\mathrm{kg})$ & Height $(\mathrm{cm})$ & Age $(\mathrm{mo})$ \\
\hline ASD group & 15 & $11.5(6.3-18.2)$ & $94(71-106)$ & $42(3-64)$ \\
Normal group & 14 & $12.9(6.3-19.2)$ & $83(63-121)$ & $24(3-70)$ \\
\hline
\end{tabular}

All data are given as mean (range). 

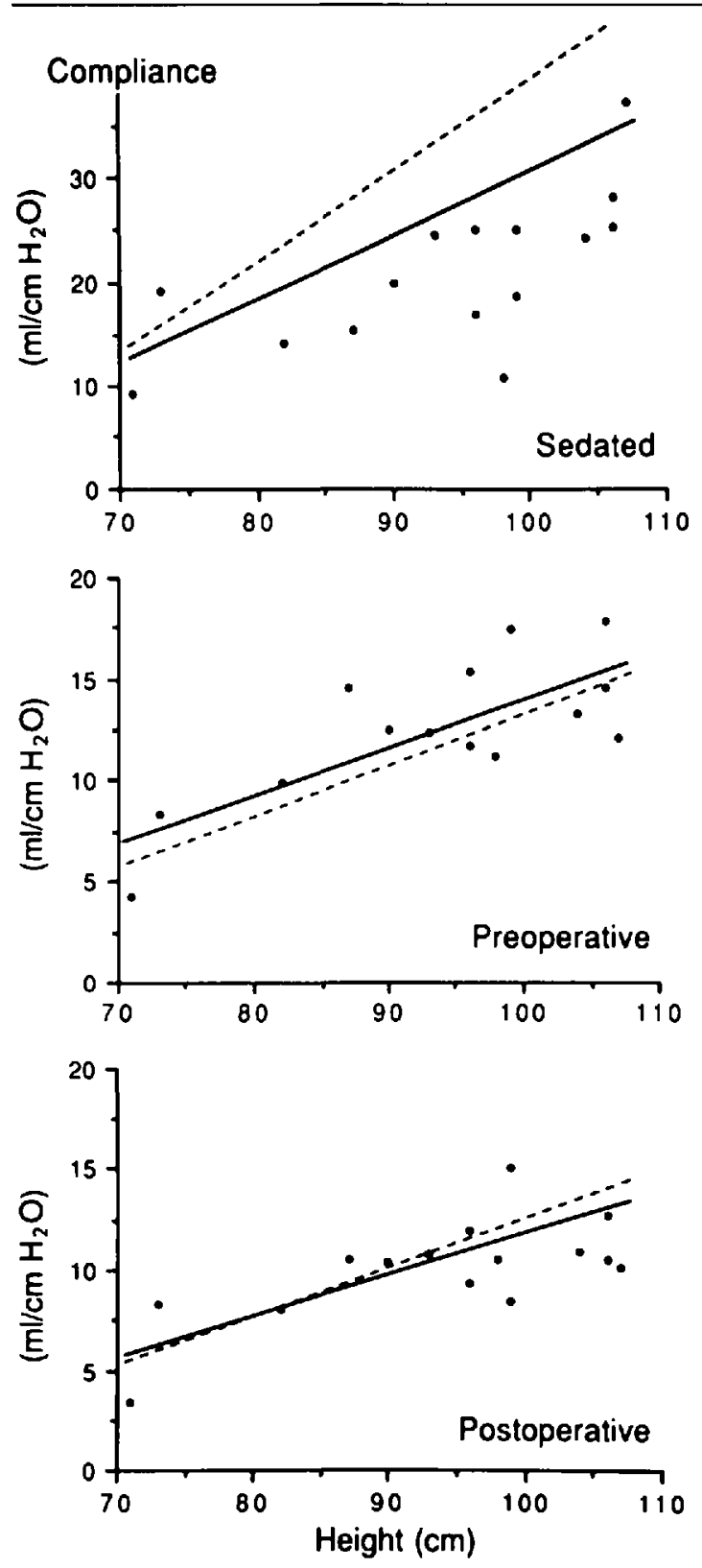

FIGURE 3 Perioperative compliance (Crs) vs height; (a) during sedation; the line of regression for the control patients is indicated by the hatched line (---) and is described by the equation $y=-36.6$ $+0.62 \times\left(r^{2}=0.85\right)$. The line of regression for the ASD patients is indicated by the solid line $(-)$ and is described by the equation $y=$ $-19.69+0.43 \times\left(r^{2}=0.46\right)$. (b) Preoperative during anaesthesia; the line of regression for the control patients is indicated by the hatched line (--) and is described by the equation $y=-12.06+0.26 \times\left(r^{2}\right.$ $=0.85$ ). The line of regression for the ASD patients is indicated by the solid line (-) and is described by the equation $y=-8.96+0.23 x$ $\left(r^{2}=0.58\right)$. (c) Postoperative during anaesthesia; the line of regression for the control patients is indicated by the hatched line (--) and is described by the equation $y=-6.77+0.19 x\left(r^{2}=0.63\right)$. The line of regression for the ASD patients is indicated by the solid line ( - ) and is described by the equation $y=-4.23+0.15 x\left(r^{2}=0.45\right)$, where $y=$ compliance in $\mathrm{ml} \cdot \mathrm{cm} \mathrm{H}_{2} \mathrm{O}^{-1}$ and $x=$ height in centimeters. difference in respiratory compliance and the Qेp:Q $\dot{Q}$ ratio $\left(\mathrm{r}^{2}=0.012, P=\mathrm{NS}\right)$ in the ASD patients studied.

\section{Discussion}

We have shown that, during sedation, Crs in children with atrial septal defects is less than Crs in normal controls. However, there is no difference in Crs between the groups during anaesthesia, either before or after surgery. This finding is unexpected in view of the fact that children in the ASD group underwent thoracic surgery involving a median sternotomy with retraction of the intra-thoracic structures and required cardiopulmonary bypass, all of which might be expected to impair respiratory mechanics further.

\section{Methodology}

In previous studies of compliance in children with congenital heart disease (CHD), the study groups were often not homogeneous. In only one study was a contemporaneous control group included but anthropometric variables of the groups were not compared. ${ }^{6}$ In the present study we have compared only children between three months and six years of age with an ASD to a matching control group. The children were chosen from the operating list in chronological order. When a child within the age range appeared on the operating list, his parents were approached for consent, and he was admitted to the study at that time, whether in the study group or control group. We could not blind the examiner to the group because the patients were studied in the appropriate operating room, making the group assignment obvious. All patients were studied by the same author (DLS). This is, to our knowledge, the first study of respiratory compliance in children with a single congenital heart lesion to be controlled in this way.

The SBT is a rapid test for measuring $\mathrm{Crs}$ in sedated children. ${ }^{10,11,18}$ The results are similar to values for $\mathrm{Crs}$ from a multiple occlusion technique in infants and children. ${ }^{13}$ However, there has not been a direct comparison of Crs by the SBT with that of the single inflation technique used here during anaesthesia. Therefore the changes observed from the sedation measurement to the anaesthetized Crs may be a result of the difference in technique. However, the use of the control measurements does allow us to examine the differences between groups in each setting, and to conclude that induction of anaesthesia seems to affect the groups differently, causing the $\mathrm{Crs}$ in the sedated normals to approach that of anaesthetized children with ASD

The SBT measurements are valid only if there is no respiratory muscle activity during the test which would interfere with the relaxed expiration after the release of the occlusion. We have previously measured abdominal wall muscle activity during the SBT with electromyography in 
sedated and anaesthetized children and found that there was no measurable abdominal wall muscle activity, which could interfere with the SBT, during sedation with chloral hydrate. ${ }^{18} \mathrm{~A}$ full discussion of respiratory muscle activity during the SBT appears in this report.

The Crs is dependent on the elastic properties of the respiratory system and on resting lung volume. We have not attempted to separate these factors by measuring specific compliance, which is Crs divided by functional residual capacity (FRC). Measuring FRC would add to the complexity of the testing and prolong the anaesthesia time. Therefore, Crs is also a measure of FRC and the changes we have described may be partially or solely due to changes in FRC.

\section{Results}

The finding that Crs during anaesthesia in the ASD group does not differ from the control group has not previously been demonstrated in a controlled study, but was suggested from studies with historical controls. Dobbinson et al. ${ }^{7}$ reported decreased specific compliance in a group of children with tetralogy of Fallot, but children with left to right intra-cardiac shunts, in their study, had intraoperative compliances similar to historical controls. Jonmarker et al. ${ }^{8}$ demonstrated compliance in children with CHD during anaesthesia but prior to surgery was similar to that obtained from an historical control group of anaesthetized children without CHD. In a conflicting study. Fisk et al. ${ }^{9}$ found intraoperative compliance of children with intrathoracic pathology was less in comparison to historical controls, but not all children had CHD in their study and some children underwent thoracotomy for non-cardiac conditions including diaphragmatic hernia.

More recently, Thorsteinsson et al. ${ }^{19}$ demonstrated that functional residual capacity in children with cardiac anomalies during anaesthesia but before surgery was not significantly different from FRC in a contemporaneous control group. Since Crs as measured by the SBT is determined, at least in part, by FRC, this finding by Thorsteinsson et al. supports the lack of a deleterious effect of anaesthesia on Crs.

In two of the previous studies of children with CHD, intraoperative compliance changes were followed serially. ${ }^{6,8}$ Jonmarker et al ${ }^{8}$ did not find a change in compliance from the beginning to the end of the surgical procedure despite the use of cardiopulmonary bypass. This finding taken with our result that no difference in $\mathrm{Crs}$ existed between the ASD group and the normal children post-surgery (Figure 3), suggests that intra-thoracic manipulation and cardiopulmonary bypass do not exert a deleterious effect on Crs. In a conflicting study, ${ }^{6}$ lung compliance decreased throughout the course of cardiac surgery in children with CHD, although specific lung compliance did not decrease. The reason for this difference in result is unclear.

The aetiology of the lesser preoperative Crs in the ASD patients compared with the control patients is not clear. There have been no observed differences in the functional residual capacity of children with congenital heart lesions compared to control patients. ${ }^{3,7}$ It has been proposed that the decreased Crs of children with increased pulmonary blood flow is caused by the increase in pulmonary blood volume. ${ }^{3}$ The lack of correlation of the echocardiographic shunt measurement with the decrease in Crs from normal in the patients with an ASD indicates that the vascular flow changes in such patients may not be solely responsible for the changes in respiratory mechanics. However, we cannot eliminate pulmonary flow changes in the aetiology of the compliance changes since the degree of pulmonary vascular changes may not be directly related to the pulmonary flow, and indeed may be inversely related to the pulmonary flow. ${ }^{20,21}$ Other factors, such as possible effects of increased respiratory infections and anatomic abnormalities on the developing lung, may also contribute to the less compliant respiratory system in patients with an ASD..$^{22.23}$

\section{Acknowledgements}

The authors wish to thank Ms. Mary Lynne MacMaster RN and the operating room Cardiovascular Nursing Team of the Hospital for Sick Children for their cooperation and assistance in this study.

\section{References}

1 Griffin AJ, Ferrara, JD, Lax JO, Cassels DE. Pulmonary compliance: an index of cardiovascular status in infancy. Am J Dis Child 1972; 123: 89-95.

2 Bancalari E, Jesse MJ, Gelband H, Garcia $O$. Lung mechanics in congenital heart disease with increased and decreased pulmonary blood flow. J Pediatr 1977; 90 192-5.

3 Howlet $G$. Lung mechanics in normal infants and infants with congenital heart disease. Arch Dis Child 1972; 47: 707-15.

4 Phelan PD, Gillam GL, Menahem SA, Coombs E, Venables $A W$. Respiratory function in infants with a ventricular septal defect. Aust Paediatr J 1972; 8: 79-85.

5 LeSouef PN, Lopes JM, England SJ, Bryan MH, Bryan $A C$. Influence of chest wall distortion on esophageal pressure. J Appl Physiol 1983; 55: 353-8.

6 Dobbinson TL, Nisbet HIA, Pelton DA, Levison H, Volgyesi $G$. Functional residual capacity (FRC) and compliance in anaesthetized paralysed children. Can Anaesth Soc J 1973; 20: 322-33. 
7 Dobbinson TL, Gray IG, Nisbet HIA, Pelton DA, Levison $H$, Volgyesi $G$. Thoracic compliance and lung volumes in children with heart discase. Acta Anaesthesiol Scand 1973; 17: 50-6.

8 Jonmarker $C$, Larsson $A$, Werner $O$. Changes in lung volume and lung-thoracic compliance during cardiac surgery in children 11 days to 4 years of age. Ancsthesiology $1986 ; 65: 259-65$.

9 Fisk GC, Deal CW. Volume pressure relations of the lungs of children measured during thoracotomy. Aust Paediatr J 1970; 6: 203-12.

10 LeSouef PN, England SJ, Bryan AC. Passive respiratory mechanics in newborns and children. Am Rev Respir Dis 1984; 129: 552-6.

11 Shulman D, Goodman A, Bar-Yishay E, Godfrey S. Comparison of the single breath and volume recruitment techniques in the measurement of total respiratory compliance in anesthetized infants and children. Anesthesiology 1989; 70: 921-7.

12 Cunningham JC, Morgan WJ, Lemen RJ, Witten ML, Margarelli JL, Quan SF. Passive exhalation technique with esophageal balloon measurements of respiratory mechanics in beagle pups. Am Rev Respir Dis 1987; 136: 722-6.

13 Guslits BG, Wilkie RA, England SJ. Comparison of methods of measurement of compliance of the respiratory system in children. Am Rev Respir Dis 1987; 136: 727-9.

14 Kitabatake A, Inoue $M$, Asao $M$ et al. Noninvasive evaluation of the ratio of pulmonary to systemic flow in atrial septal defect by duplex Doppler echocardiography. Circulation 1984; 69: 73-9.

15 Christie J, Sheidahl LM, Tristani FE, Sagar KB, Ptacin $M J$, Wann $S$. Determination of stroke volume and cardiac output during exercise: comparison of twodimensional and Doppler echocardiography, Fick oximetry, and thermodilution. Circulation 1987; 76: 539-47.

16 Goldberg SJ, Sahn DJ, Allen HD, Valdes-Cruz LM, Hoenecke H, Carnahan $Y$. Evaluation of pulmonary and systemic blood flow by 2-dimensional doppler echocardiography using fast fourier transformed spectral analysis. Am J Cardiol 1982; 50: 1394-400.

17 Valdes-Cruz LM, Horowitz S, Mesel E, Sahn DJ, Fisher $D C$, Larson D. QA pulsed doppler echocardiographic method for calculating pulmonary and systemic blood flow in atrial level shunts: validation studies in animals and initial human experience. Circulation 1984; 69: 80-6.

18 Shulman $D L$, Volgyesi GA, Burrows FA, Lerman J, England SJ. Expiratory muscle activity in anesthetized children: effect on the single breath technique. Pediatr Pulmon 1989; 7: 82-8.
19 Thorsteinsson A, Jonmarker C, Larsson A, Vilstrup C. Werner $O$. Functional residual capacity in ancsthetized children: normal values and values in children with cardiac anomalies. Ancsthesiology 1990; 73: 876-81.

20 Burrows $F A$, Rabinovitch $M$. The pulmonary circulation in children with congenital heart disease: morphologic and morphometric considerations. Can Anacsth Soc J 1985, 32 : 364-73

21 Burrows FA, Klinck JR, Rabinovitch M, Bohn DJ. Pulmonary hypertension in children: perioperative management. Can Anaesth Soc J 1986; 33: 606-28.

22 Rudolph AM. Pulmonary complications of congenital heart disease. Pediatrics 1969; 43: 757-8.

23 Stanger $P$, Lucas $R V J r, E d w a r d s J E$. Anatomic factors causing respiratory distress in acyanotic congenital cardiac disease. Pediatrics 1969; 43: 760-9. 